\title{
Preparation and evaluation of chitosan-tripolyphosphate nanoparticles suspension as an antibacterial agent
}

\author{
Hakim Bangun*, Steven Tandiono, Anayanti Arianto \\ Department of Pharmaceutical Technology, Faculty of Pharmacy, Nanomedicine Centre of Innovation, University of Sumatera Utara, Jl. Tri Dharma No. 5, \\ Kampus USU, Medan, Indonesia.
}

\section{ARTICLE INFO \\ Received on: 26/07/2018 \\ Accepted on: 11/11/2018 \\ Available online: $30 / 12 / 2018$}

Key words:

Chitosan, sodium

tripolyphosphate,

nanoparticles, antibacterial activity.

\begin{abstract}
Objectives: The purpose of this study was to prepare and evaluate the chitosan-tripolyphosphate (CS-TPP) nanoparticles suspension as an antibacterial agent.

Material and Methods: CS-TPP nanoparticles suspension was prepared by the ionic gelation method with various concentrations of CS and TPP. The prepared nanoparticles suspension was evaluated for particle size, sedimentation, the interaction between CS and TPP, particles morphology, and antibacterial activity against Staphylococcus aureus (Gram positive) and Pseudomonas aeruginosa (Gram negative).

Results: The results showed that among all of the concentrations used, the formulation of $0.25 \% \mathrm{CS}$ and $0.1 \%$ sodium TPP resulted in the smallest particles size (average diameter was $238.17 \mathrm{~nm}$ ) with spherical morphology, no sedimentation after centrifugation at $6,000 \mathrm{rpm}$ for 2 hours, and gave the highest antibacterial activity against Staphylococcus aureus and P. aeruginosa.

Conclusion: CS-TPP nanoparticles can be formed by using CS and TPP solution at a certain concentration and the CS-TPP nanoparticles suspension has an antibacterial activity.
\end{abstract}

\section{INTRODUCTION}

Nanoparticles are defined as a particulate dispersion or solid particles with a size in the range of 10-1,000 nm (Kayser et al., 2005). Nanoparticles can consist of different biodegradable materials like natural or synthetic polymers, lipid, or phospholipids (Kayser et al., 2005). Polymeric nanoparticles offer some specific advantages over liposome, for instance, they increase the stability of drug and possess useful controlled release properties (Calvo, et al., 1997a). Nanoparticulate systems show their promise as a potential ideal drug delivery system for poorly soluble, poorly absorbed, and chemically, heat- and photo-labile substances (Florence, 1998). The nanoparticles system offer advantages like increased bioavailability, site-specific drug delivery, sustained release of the

*Corresponding Author

Hakim Bangun, Department of Pharmaceutical Technology, Faculty of Pharmacy, Nanomedicine Centre of Innovation, University of Sumatera Utara, Jl. Tri Dharma No. 5, Kampus USU, Medan, Indonesia.

E-mail: hakimb17@yahoo.com drug over a longer period of time, and increased patient compliance due to a reduction in frequent dosing (Chen et al., 2001).

Chitosan (CS) is a modified natural carbohydrate polymer prepared by the partial $\mathrm{N}$-deacetylation of chitin, a natural biopolymer derived from crustacean shells such as crabs, shrimps, and lobster (Illum, 1998). CS is available in a wide range of molecular weight and degree of deacetylation. Molecular weight and degree of deacetylation are the main factors affecting the particle size, particles formation, and aggregation (Singh et al., 2015). CS entraps drugs through a number of mechanisms, including ionic cross-linking (Prabaharan and Mano, 2005). CS has been used as a drug delivery system (Arianto et al., 2014; 2015; Mariadi, 2015). CS exhibits many advantages in developing nanoparticles, including biocompatibility, biodegradability, and low immunogenicity. The high positive charge density also confers its mucoadhesive properties and makes it an ideal candidate for the delivery of drugs to mucosal tissues. CS also has a very low toxicity (Nagarajan et al., 2015).

Sodium tripolyphosphate (TPP) is known as crosslinking agents. Shu and Zhu (2002) reported the use of sodium TPP for CS 
gel formation. Sodium TPP can improve the mechanical strength of the gel formed. This is because sodium TPP has a high negative charge density to interact with polycationic CS. According to Yongmei and Yumin (2003), the formation of nanoparticles occurs only at a certain concentration of CS and TPP. The role of TPP as a crosslinking agent will strengthen the matrix of the CS nanoparticles. With the increasing number of crosslinks formed between CS and TPP, the mechanical strength of the CS matrix will increase so that CS particles become stronger and harder, and more difficult to split into smaller parts (Yongmei and Yumin, 2003).

$\mathrm{CS}$, as a cationic natural polymer, has been used widely as an antimicrobial agent for preventing and treating infectious disease. It is due to its intrinsic antimicrobial properties and its ability to deliver the antimicrobial compound to the infected area (Dai et al., 2011; Kong et al., 2010). CS inhibited and suppressed microbial activities through their electrostatic charge interaction between positive charges on polycationic CS molecules (amino groups) with negative charges on the microbial surface (Aziz et al., 2012). This interaction caused disruption of the microbial cells, which then changed their metabolism and led to cell death (Leceta et al., 2013).

CS can form nanoparticles using various methods, one of them is the ionic gelation method (Agnihotri et al., 2004; Antoniou et al., 2015). This ionic gelation method is widely used in the formation of CS nanoparticles (Calvo et al., 1997; Kumar et al., 2011; Qi et al., 2004), where the positively charged amino group of CS interacts with the negative charged TPP to form coacervates with a size in the range of nanometer (Calvo et al., 1997; Kumar et al., 2011; Qi et al., 2004). Coacervates are formed as a result of electrostatic interaction between two aqueous phases, whereas ionic gelation involves the material undergoing the transition from liquid to gel due to ionic interaction conditions at room temperature (Mohanraj and Chen, 2006).

In a previous study conducted by Calvo et al. (1997), CS-TPP nanoparticles have been formulated by the ionic gelation method. However, that study did not show the evaluation of the CS nanoparticles antibacterial activity. In other studies conducted by Kumar et al. (2011) and Ibrahim et al. (2015), the formulation and evaluation of antibioticsloaded nanoparticles have been done by ionic gelation methods. Another study conducted by Qi et al. (2004) has been performed on the preparation of CS-TPP nanoparticles and copper-loaded CS-TPP nanoparticles. In that study, the evaluation of the antibacterial activity of CS-TPP nanoparticles and copperloaded nanoparticles have been investigated and showed that CS-TPP nanoparticles have an antibacterial activity. However, they did not study the effect of particle size of CS-TPP on the antibacterial activity.Therefore, this study will discuss the preparation of CS-TPP nanoparticles suspension by the ionic gelation method and study the effect of particle size of CS-TPP on the antibacterial activity. Besides, the antibacterial activity of CS-TPP nanoparticle and the CS solution without sodium TPP crosslinking was compared.

\section{MATERIALS AND METHODS}

\section{Material}

CS (Funakoshi Co., Ltd), glacial acetic acid (Merck), sodium TPP, Staphylococcus aureus (SA ATCC 6538),
Pseudomonas aeruginosa (PA ATCC 9027), Muller Hinton agar (MHA), nutrient broth, and nutrient agar.

\section{Methods}

Preparation of CS-TPP nanoparticles suspension with various of CS concentrations

CS $(0.05,0.1,0.15$, and $0.2 \mathrm{~g})$ was dissolved in $20 \mathrm{ml}$ of $1 \%(\mathrm{v} / \mathrm{v})$ acetic acid and leaving it under stirring for 8 hours. Thereafter, they were sonicated for 40 minutes. TPP $0.01 \mathrm{~g}$ was dissolved separately in $10 \mathrm{ml}$ distilled water and leaving it under stirring for 8 hours. Then, it was sonicated for 40 minutes. TPP solution was then added to CS solution dropwise using a $1 \mathrm{ml}$ syringe (drip rate $15 \mathrm{drops} /$ minute) until CS:TPP ratio $=2: 1$. Subsequently, this solution was stirred for another 8 hours and sonicated for 45 minutes.

Preparation of nanoparticles suspension with various of TPP concentrations

Preparation of nanoparticles suspension with a variation in TPP concentrations was done with the same procedure with the variation of CS concentrations, but using the constant $0.5 \%$ of CS concentration and with the variation of TPP concentrations $(0.1 \%$, $1 \%$, and $2 \%$ ).

\section{Evaluation of particles size}

Particle size analysis of CS-TPP nanoparticles was performed by using CORDOUAN Technologies Particle Size Analyzer at room temperature.

\section{Evaluation of suspension sedimentation}

Evaluation of CS-TPP nanoparticles suspension was performed by using a centrifuge (Hitachi CF16RXII). The samples were filled in centrifuge tubes, thereafter, the centrifugation was done at 6,000 rpm for 2 hours. Then, the sediment was observed. If there were no sedimentation, it means the suspension has a particle size in a range of nanometer.

\section{Microscopic characterization of particles}

Microscopic characterization of particles was done to investigate the morphology of particles by using a microscope (Nikkon). This microscopic evaluation was performed by using sediment after centrifugation of the nanoparticles suspension. The sediment was placed on object glass and was added with a small drop of methylene blue as a coloring agent. This preparate was covered with a cover glass. Then, the observation was made using a $10 \times$ magnification lens.

\section{TEM analysis}

Shape and size of nanoparticles were investigated using Transmission Electron Microscopy (TEM) (JEOL JEM 1400). This evaluation was performed on nanoparticles suspension with the smallest particle size among all nanoparticle formulations.

\section{FT-IR analysis}

Fourier Transform Infra Red (FT-IR) (Shimadzu) analysis was performed on CS powder, TPP powder, and sediment of nanoparticles using $\mathrm{KBr}$ pellets in the range of $500-4,000 \mathrm{~cm}^{-1}$. 


\section{Evaluation of antibacterial activity}

The evaluation was performed using Staphylococcus aureus and Pseudomonas aeruginosa as the model of Gram-positive and Gram-negative bacteria, respectively, on MHA media. Where as much as $0.1 \mathrm{ml}$ of inoculums were placed in a Petri dish, then added to $15 \mathrm{ml}$ of sterile MHA media, homogenized, and waited until the media were solidified. Then, with the help of the sterile cork borer, four wells were created in all the MHA plates. The wells were labeled and loaded each with $25 \mu$ of CS-TPP nanoparticles suspension and $\mathrm{CS}$ solution. The plates were incubated at $36^{\circ} \mathrm{C}-37^{\circ} \mathrm{C}$ for 24 hours and observed for the diameter of Zone of Inhibition (ZOI) around the wells and were measured. This test was repeated three times.

\section{RESULTS AND DISCUSSION}

\section{Preparation of CS-TPP nanoparticles suspension}

Preparation of CS-TPP nanoparticles suspension was carried out based on a study done by Calvo et al. (1997), that was based on the ionic gelation method. Calvo et al. formulated CS-TPP nanoparticles with $0.05 \%, 0.1 \%, 0.5 \%$, and $1 \%$ CS concentrations in acetic acid. The concentration of acetic acid used was as much as 1.75 times higher than that of CS concentration. The TPP concentration used was $0.05 \%, 0.1 \%, 0.5 \%$, and $1 \%$. Then, a variable volume of TPP solution $(0.25,1,2,2.5$, and $3 \mathrm{ml})$ was added to $5 \mathrm{ml}$ of the $\mathrm{CS}$ solution under magnetic stirring at room temperature.

In the present study, CS-TPP nanoparticles suspension was also formulated using the ionic gelation method. This method involves ionic interactions between the positive charge of CS with the negative charge of TPP. CS solution was prepared by dissolving CS in $1 \%$ acetic acid. This dissolution of CS was done by using a magnetic stirrer at low speed for 8 hours. TPP solution was prepared by dissolving a certain amount of TPP in distilled water using a low-speed magnetic stirrer for 8 hours. Then, both of these polyelectrolyte solutions were sonicated for 40 minutes to complete their solubility. TPP solution was then added to CS solution dropwise at a drip rate of $15 \mathrm{drops} /$ minute while still being stirred at $100 \mathrm{rpm}$, then this solution was stirred again for another 8 hours and sonicated for 45 minutes to complete the dispersion of nanoparticles.

When TPP is added to the CS solution, cross-linking of the negative charge of TPP with the positive charge of CS occurs. This interaction will result in the formation of a nano-sized polyelectrolyte complex with the help of stirring and sonication. Based on the research of Calvo et al. (1997), it shows that the concentration of each solution will affect the particle size of the complex. The increasing concentration of CS will increase the size of the particles formed. This also occurs in the increasing concentration of TPP.
In this present study, CS-TPP nanoparticles suspension was prepared with $0.25 \%, 0.5 \%, 0.75 \%$, and $1 \%$ CS concentrations and also with $0.1 \%, 1 \%$, and 2\% TPP concentrations. Effect of various concentrations of these two solutions was then evaluated against the particle size and the antibacterial activity.

\section{Effect of CS concentrations on CS-TPP nanoparticles size}

Effect of CS concentrations $(0.25 \%, 0.5 \%, 0.75 \%$, and $1 \%$ ) on particle size was evaluated by using a particle size analyzer. The result showed that with increasing CS concentration from $0.25 \%$ to $1 \%$ and with the constant of $0.1 \%$ TPP concentration, the particle size of nanoparticles suspension increased as shown in Table 1. Similar results were also obtained from the research of Calvo et al. (1997), which stated that the minimum size $(260 \mathrm{~nm})$ was obtained from the lowest CS concentration $(1 \mathrm{mg} / \mathrm{ml})$ and the particle size increases with the increasing CS concentration.

\section{Effect of TPP concentrations on CS-TPP particle size}

The effect of TPP concentrations $(0.1 \%, 1 \%$, and $2 \%)$ was observed in constant $0.5 \% \mathrm{CS}$ concentration. TPP was a major ingredient for crosslinking and has a pronounced effect on the formation of nanoparticles suspension. The increasing concentration of TPP that was used, above $0.1 \%$, resulted in precipitation or sedimentation in the solution. Precipitation indicates the larger particle size in the solution. The precipitation at an excessive higher concentration of TPP may be due to the interaction between CS and TPP achieved stoichiometry and resulted in more the amount of CS-TPP complexes formed. The precipitated substance was proven by FTIR analysis as shown in the evaluation of the FTIR analysis below (Fig. 9). In this complexation, 1 monomer of TPP interacts with 2 monomers of CS (Ibrahim et al., 2015).As shown in Fig. 1 that with the $0.1 \%$ concentration of TPP, the particles were still homogenously dispersed and no precipitation exists. However, with the $1 \%$ and $2 \%$ TPP concentrations, precipitation occurred immediately. However, if the suspended suspension was re-stirred, then the particles easily be dispersed again.

\section{Evaluation of suspension sedimentation}

The result of CS-TPP nanoparticles suspension sedimentation test after centrifugation at $6,000 \mathrm{rpm}$ for 2 hours with $0.25 \%, 0.5 \%, 0.75 \%$, and $1 \%$ of CS concentrations with $0.1 \%$ of TPP concentration can be seen in Figure 2 and with $0.5 \%$ of CS concentration and $0.1 \%, 1 \%$, and $2 \%$ of TPP concentrations can be seen in Figure 3.

The result showed that the precipitation did not occur in nanoparticles suspension with $0.25 \%$ and $0.5 \%$ CS concentrations. However, sedimentation occurred in nanoparticles suspension with $0.75 \%$ and $1 \%$ concentration of CS. As for the suspension with the variation of TPP can be seen that for the suspension with $1 \%$ and

Table 1. Effect of CS concentrations at $0.1 \%$ sodium TPP solution on the particle size of CS-TPP nanoparticles suspension formed.

\begin{tabular}{cccc}
\hline No. & CS concentrations $(\%)$ & Particle size of CS-TPP $(\mathbf{n m})$ & Range of particle size $(\mathbf{n m})$ \\
\hline 1 & 0.25 & 238.17 & $89.15-513.00$ \\
2 & 0.50 & 575.20 & $223.93-1230.59$ \\
3 & 0.75 & 706.01 & $257.11-1549.23$ \\
4 & 1 & 1315.37 & $467.86-2951.99$ \\
\hline
\end{tabular}




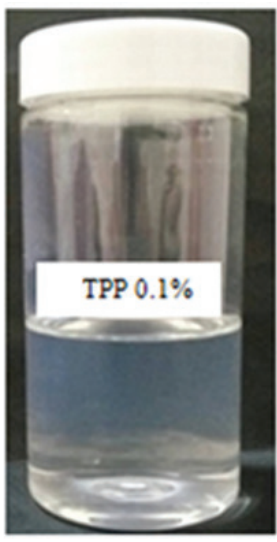

a

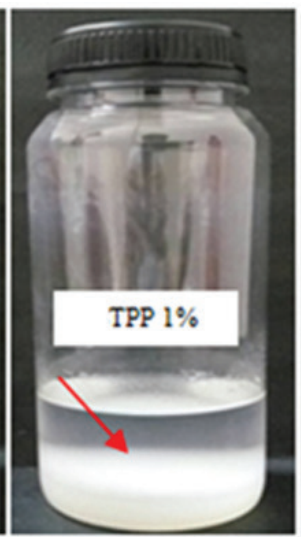

$\mathrm{b}$

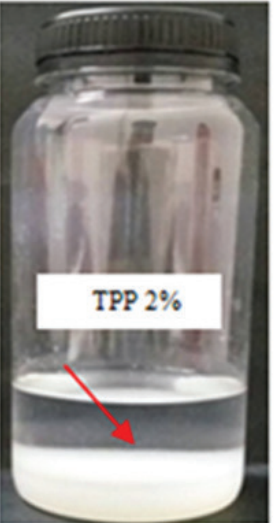

C

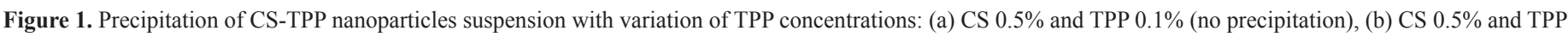
$1 \%$ (precipitation), and (c) CS $0.5 \%$ and TPP $2 \%$ (precipitation). Arrow shows precipitation.

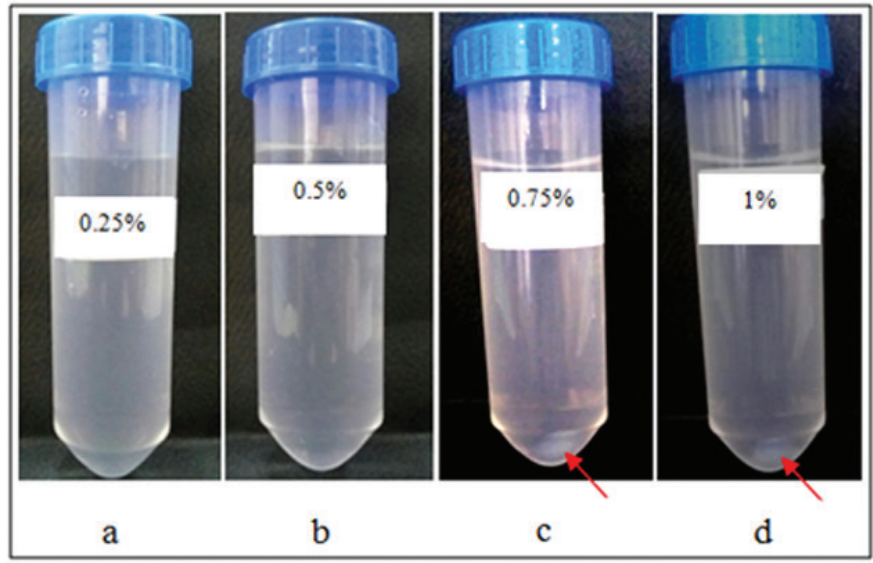

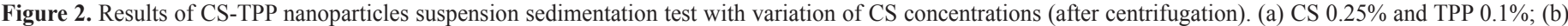
CS $0.50 \%$ and TPP $0.1 \%$; (c) CS $0.75 \%$ and TPP $0.1 \%$; and (d) CS $1 \%$ and TPP $0.1 \%$. Arrow shows sedimentation. (a and b) No sedimentation.

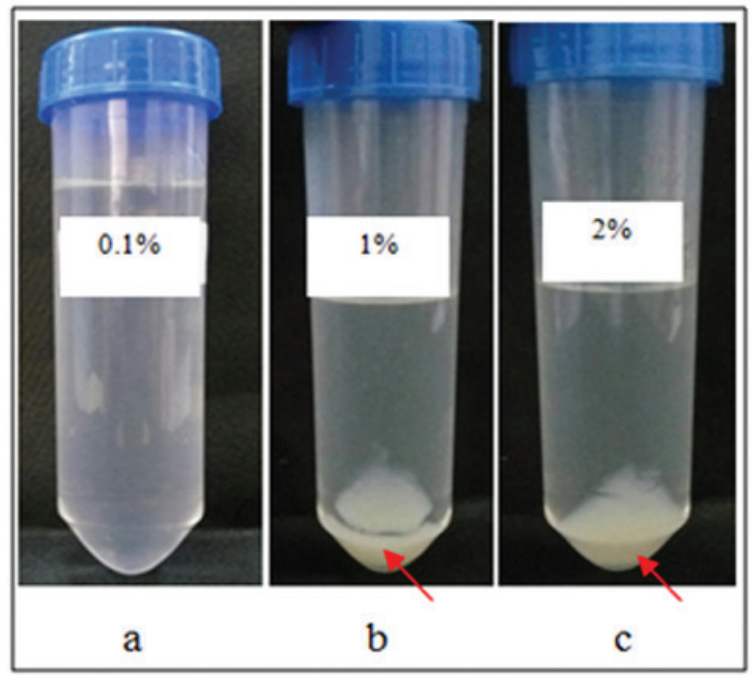

Figure 3. Results of CS-TPP suspension sedimentation test with variation of TPP concentrations (after centrifugation). (a) CS $0.5 \%$ and TPP $0.1 \%$; (b) CS $0.5 \%$ and TPP $1 \%$; and (c) CS $0.5 \%$ and TPP $2 \%$. Arrow shows sedimentation. (a) No sedimentation. 
$2 \%$ TPP concentrations, precipitation occurred. This is due to the nanoparticles suspension with $0.25 \%$ and $0.5 \%$ CS concentrations, the particle size contained in the suspension is smaller than that in the suspension with $0.75 \%$ and $1 \%$ CS concentrations and the suspension with $1 \%$ and $2 \%$ TPP concentrations. The smaller the particle size, the sedimentation rate will be smaller, therefore, at the same centrifugation rate and the same centrifugation time, the suspension with a much smaller particle size will have a much lower sedimentation rate compared to the suspension with larger particle size.

\section{Microscopic view of particles}

Microscopic view of sediment after centrifugation of $0.75 \%$ and $1 \%$ of CS concentrations with $0.1 \%$ of TPP concentration can be seen in Figure 4 and that for $0.5 \%$ of CS concentration and $1 \%$ and $2 \%$ with TPP concentrations can be seen in Figure 5.

\section{TEM images}

The morphology of CS-TPP nanoparticles suspension observed by using TEM is shown in Figure 6. Figure 6 shows that nanoparticles suspension with $0.25 \% \mathrm{CS}$ and $0.1 \%$ TPP is a spherical particle.

\section{FT-IR analysis}

FT-IR analysis was performed on CS powder, TPP powder, and sediment of nanoparticles with $0.75 \% \mathrm{CS}$ and $0.1 \%$ TPP. The results can be seen in Figures 7-9.

From Figure 7, we can see the FT-IR spectrum of CS that shows absorption peak at $3,421.72 \mathrm{~cm}^{-1}$ is assigned to $\mathrm{OH}$ group and $\mathrm{NH}_{2}$ group; $2,924.1 \mathrm{~cm}^{-1}$ that shows absorption bands of $\mathrm{C}-\mathrm{H} ; 1,639.49 \mathrm{~cm}^{-1}$ shows absorption bands of $\mathrm{C}=\mathrm{O}$ from amide group of CS; $1,554.63 \mathrm{~cm}^{-1}$ has been assigned to $\mathrm{N}-\mathrm{H} ; 1,373.32 \mathrm{~cm}^{-1}$ shows the stretching vibration of $\mathrm{C}-\mathrm{H}$ from $\mathrm{CH}_{2} \mathrm{OH}$ groups; $1,072.42 \mathrm{~cm}^{-1}$ shows stretching vibration of $\mathrm{C}-\mathrm{O}$ from $\mathrm{CH}-\mathrm{OH}$; and $983.7 \mathrm{~cm}^{-1}$ shows stretching vibration of $\mathrm{C}-\mathrm{O}$ from $\mathrm{CH}_{2} \mathrm{OH}$ (Nie et al., 2015; Yasmeen et al., 2016). Whereas, the FT-IR spectra of TPP can be observed in Figure 8 that shows absorption bands at $1,211.30-1,087.85 \mathrm{~cm}^{-1}$ is assigned to $\mathrm{P}=\mathrm{O}$ groups and bands at $894.97 \mathrm{~cm}^{-1}$ is assigned to $\mathrm{P}-\mathrm{O}$ (Jafary et al., 2016; Nie et al., 2015).

Figure 9 shows FTIR spectrum of CS-TPP nanoparticles that have a similar spectrum with CS but there is a difference at $3,421.72 \mathrm{~cm}^{-1}$ that is broadened due to the interaction of phosphate group from TPP with $\mathrm{NH}_{2}$ group from $\mathrm{CS} ; 1,539.20 \mathrm{~cm}^{-1}$ shows that there is a difference in $\mathrm{N}-\mathrm{H}$ group and also a difference in the absorption bands at $1,076.28-902.69 \mathrm{~cm}^{-1}$ due to $\mathrm{P}=\mathrm{O}$ and $\mathrm{P}-\mathrm{O}$ groups of TPP. The overlapping FT-IR spectrum of the three compounds can be seen in Figure 10.

\section{Antibacterial activity}

This antibacterial activity test was performed on Grampositive bacteria and Gram-negative bacteria. Gram-positive bacteria used in this study were Staphylococcus aureus. Whereas, Gram-negative bacteria used were Pseudomonas aeruginosa. The test method used was the agar diffusion method.

Results of antibacterial activity test from CS solution and nanoparticle suspension with $0.25 \%, 0.5 \%, 0.75 \%$, and $1 \%$ CS concentrations against the growth of Staphylococcus aureus and Pseudomonas aeruginosa are listed in Tables 2 and 3 and Figures 11-14.

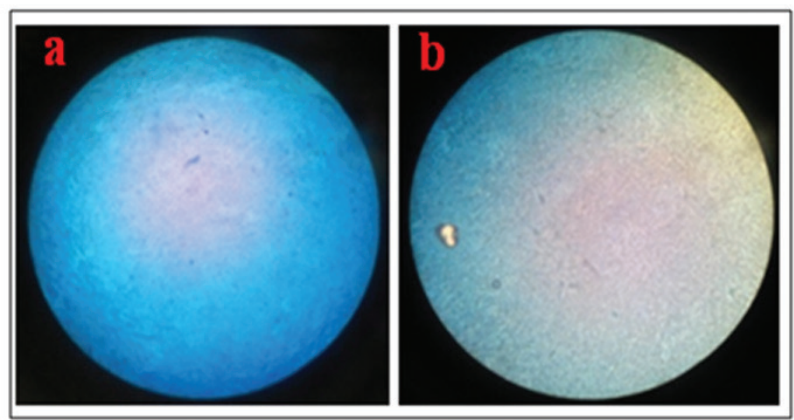

Figure 4. Microscopic view of nanoparticles sediment with variation of CS concentrations. (a) CS $0.75 \%$ and TPP $0.1 \%$ and (b) CS $1 \%$ and TPP $0.1 \%$ ( $10 \times$ magnification).

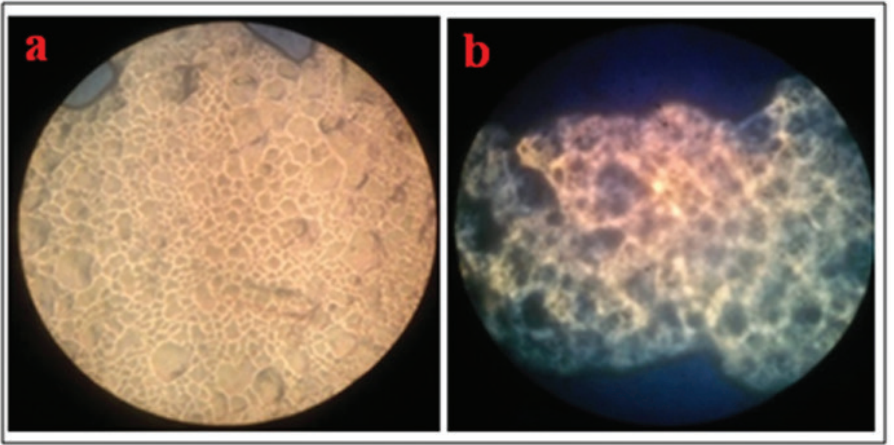

Figure 5. Microscopic view of sediment with variation of TPP concentrations. (a) CS $0.5 \%$ and TPP $1 \%$ and (b) CS $0.5 \%$ and TPP $2 \%$ (10× magnification). 

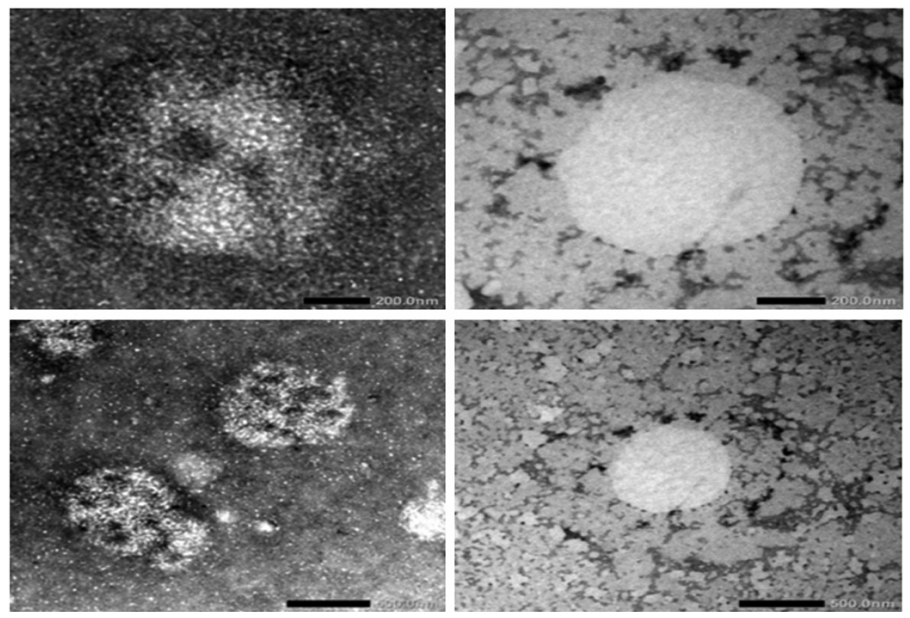

Figure 6. TEM images of nanoparticles suspension of CS-TPP prepared from $0.25 \%$ CS with $0.1 \%$ TPP.

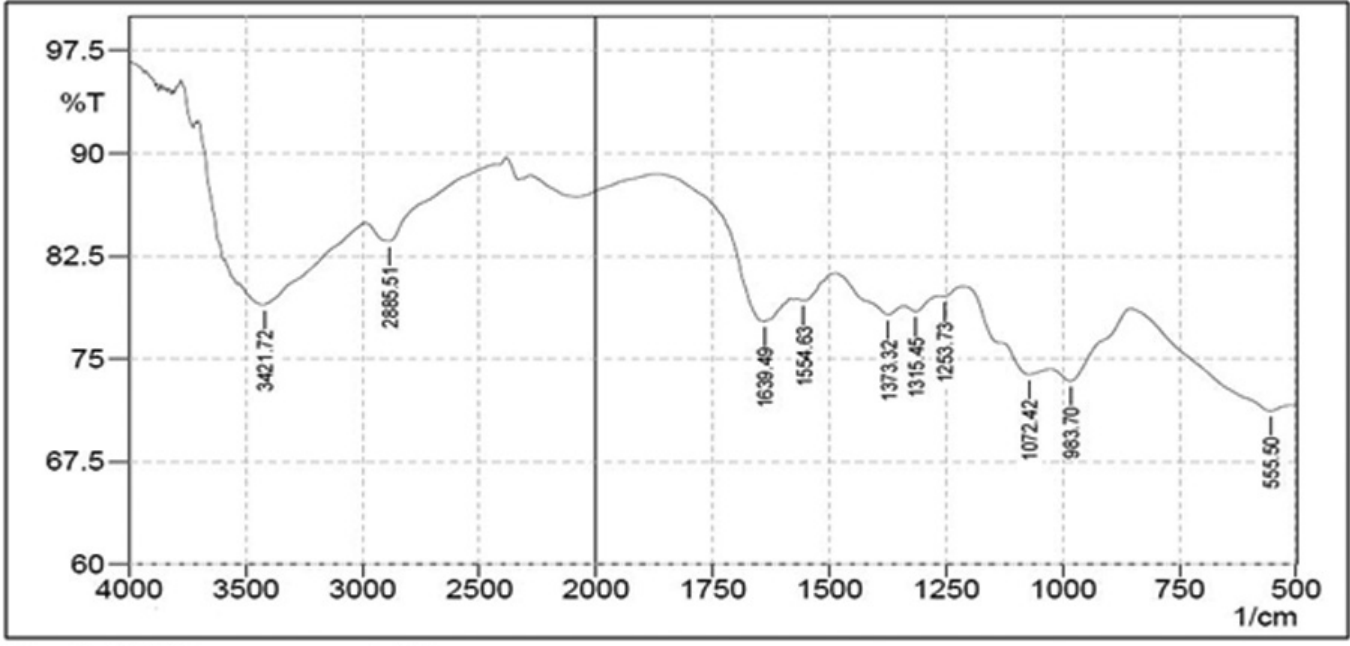

Figure 7. FT-IR spectrum of CS.

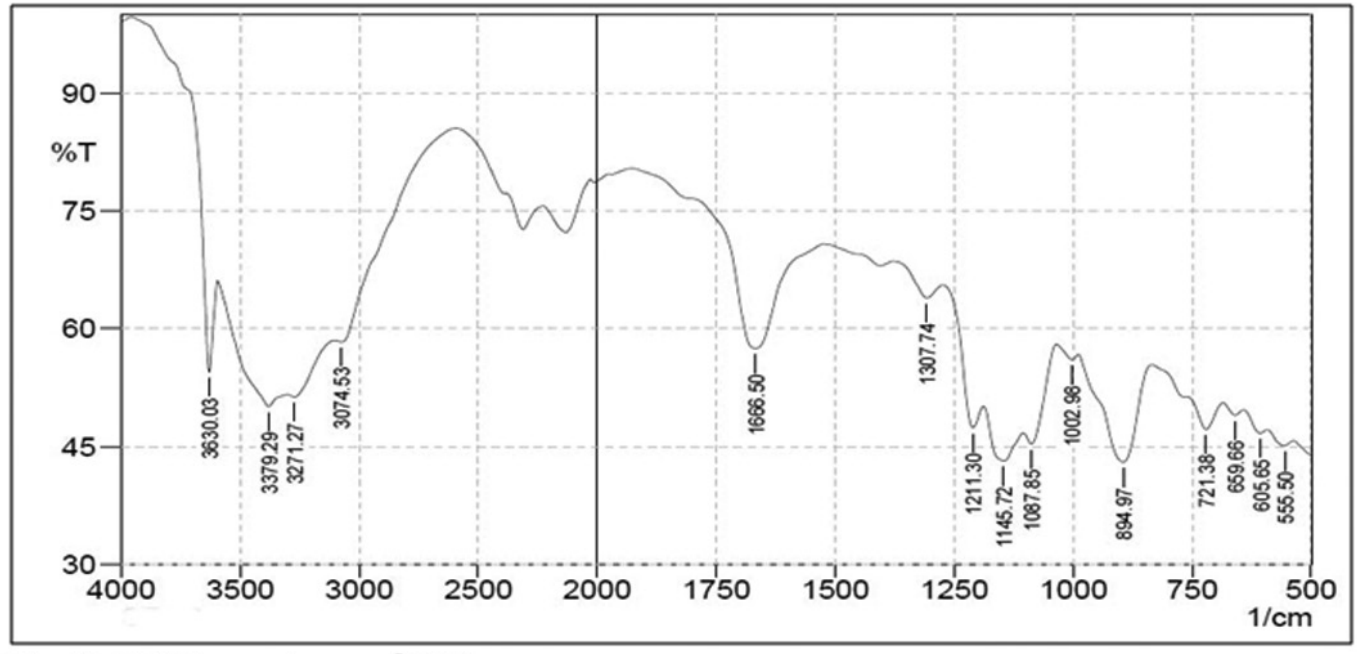

Figure 8. FT-IR spectrum of TPP. 


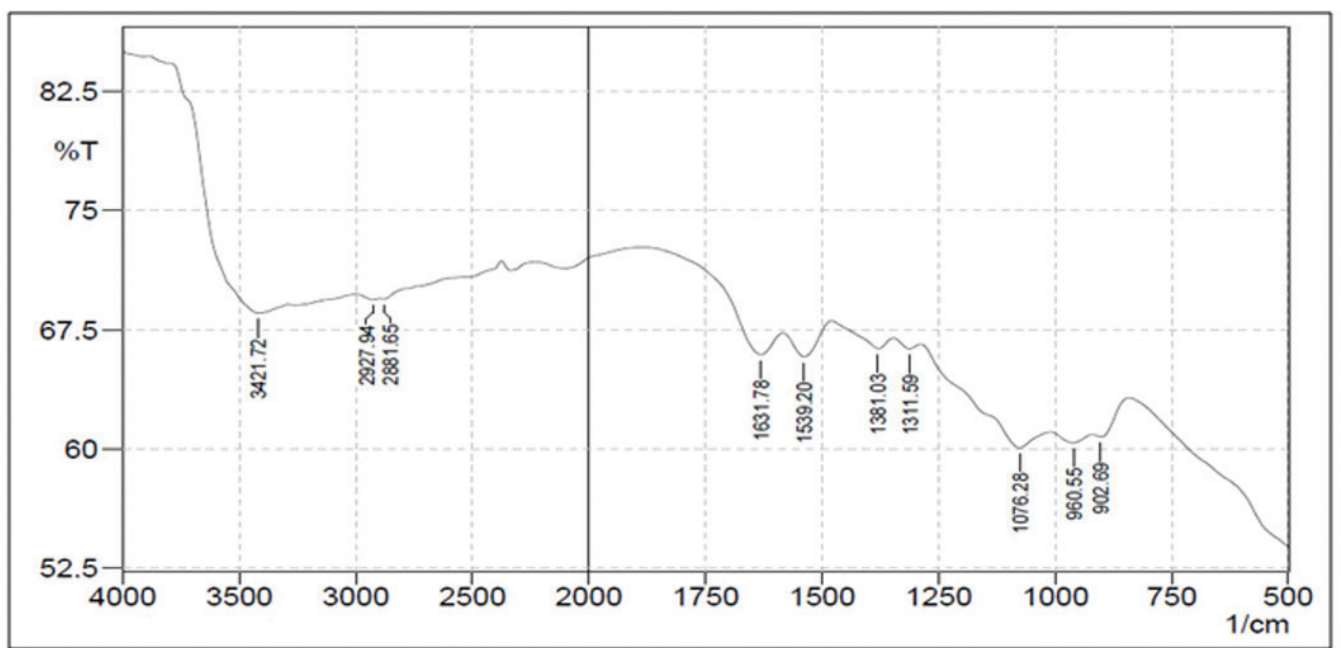

Figure 9. FT-IR spectrum of CS-TPP nanoparticles.

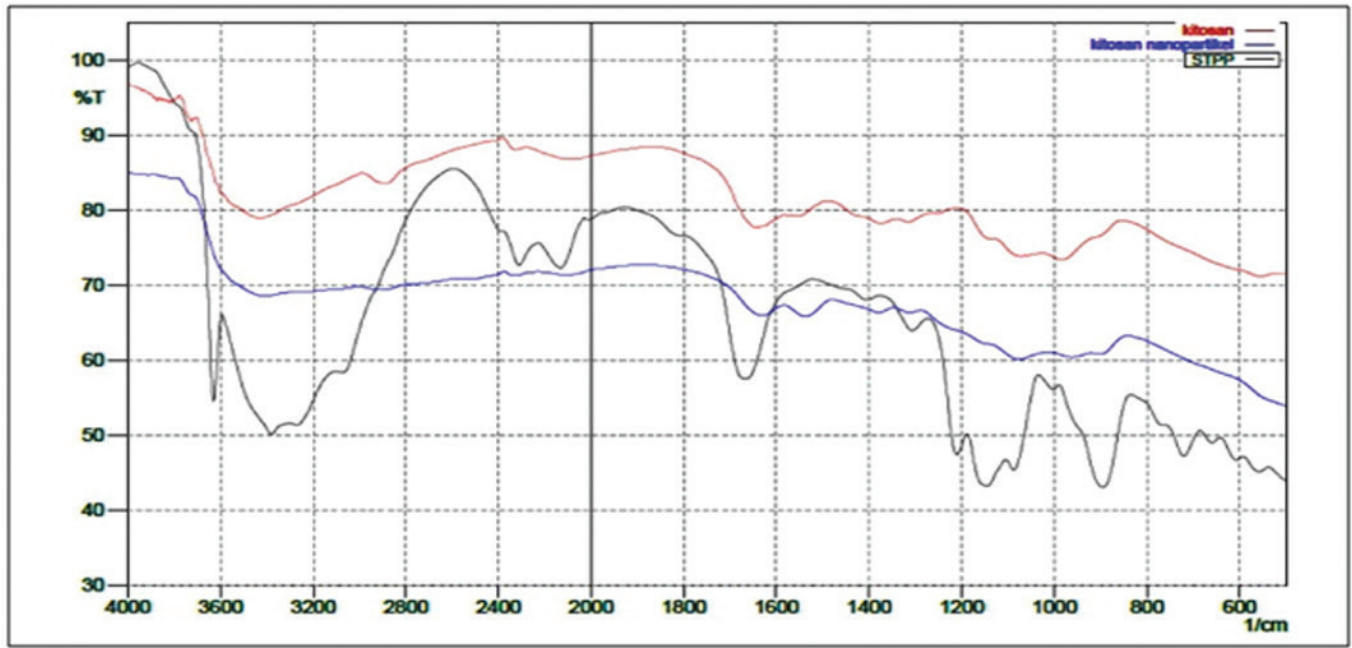

Figure 10. Overlapping FTIR spectra of CS, TPP, and nanoparticles.

Table 2. Diameter ZOI of CS solution and CS-TPP nanoparticles against Staphylococcus aureus.

\begin{tabular}{cccc}
\hline No & CS concentration (\%) & TPP concentration (\%) & Diameter ZOI (mm) \\
\hline 1 & 0.25 & - & $8.83 \pm 0.21^{*}$ \\
2 & 0.50 & - & $9.42 \pm 0.25^{*}$ \\
3 & 0.75 & - & $10.03 \pm 0.41^{*}$ \\
4 & 1 & - & $10.53 \pm 0.20^{*}$ \\
5 & 0.25 & 0.1 & $12.42 \pm 0.21^{* *}$ \\
6 & 0.50 & 0.1 & $11.52 \pm 0.18^{* *}$ \\
7 & 0.75 & 0.1 & $11.07 \pm 0.19^{* *}$ \\
8 & 1 & 0.1 & $10.75 \pm 0.13^{* *}$ \\
\hline
\end{tabular}

Note: * CS solution; ** CS-TPP nanoparticles. 
Table 3. Diameter ZOI of CS solution and CS-TPP nanoparticles against Pseudomonas aeruginosa.

\begin{tabular}{cccc}
\hline No & CS concentration (\%) & TPP concentration (\%) & Diameter ZOI (mm) \\
\hline 1 & 0.25 & - & $8.77 \pm 0.55^{*}$ \\
2 & 0.50 & - & $9.38 \pm 0.30^{*}$ \\
3 & 0.75 & - & $10.02 \pm 0.28^{*}$ \\
4 & 1 & - & $10.47 \pm 0.29^{*}$ \\
5 & 0.25 & 0.1 & $11.9 \pm 0.26^{* *}$ \\
6 & 0.50 & 0.1 & $11.3 \pm 0.20^{* *}$ \\
7 & 0.75 & 0.1 & $10.85 \pm 0.13^{* *}$ \\
8 & 1 & 0.1 & $10.47 \pm 0.20^{* *}$ \\
\hline
\end{tabular}

Note: * CS solution; ** CS-TPP nanoparticles.

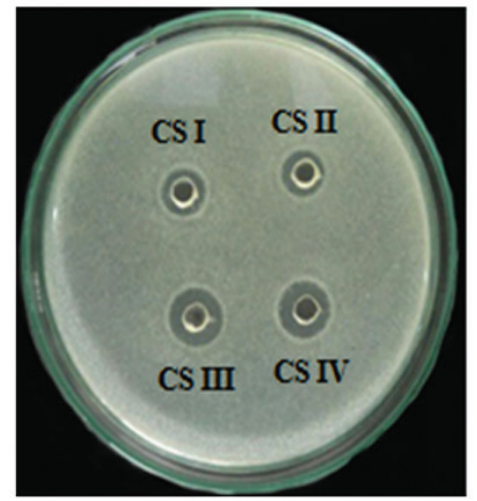

CS I : CS $0.25 \%$ solution

CS II : CS $0.50 \%$ solution

CS III : CS $0.75 \%$ solution

CS IV : CS $1 \%$ solution

Figure 11. Antibacterial activity of CS solutions against Staphylococcus aureus.

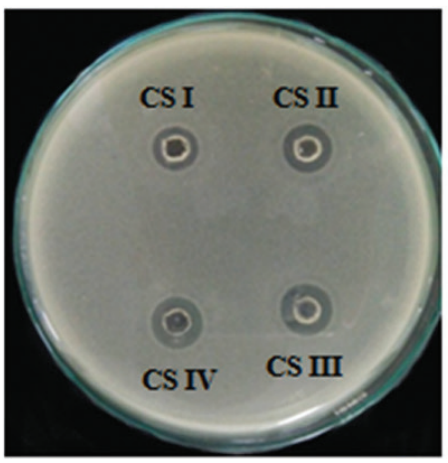

CS I : CS $0.25 \%$ solution

CS II : CS $0.50 \%$ solution

CS III : CS $0.75 \%$ solution

CS IV : CS $1 \%$ solution

Figure 12. Antibacterial activity of CS solutions against Pseudomonas aeruginosa.

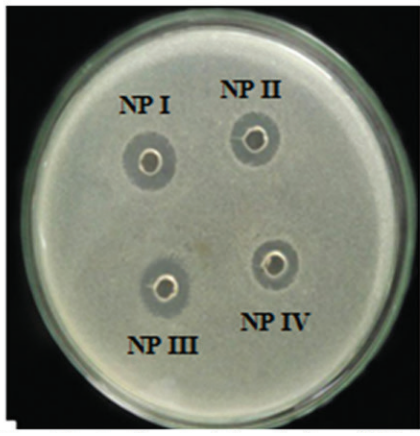

NPI : Nanoparticles of CS-TPP $(0.25 \%$ and $0.1 \%)$

NPII : Nanoparticles of CS-TPP $(0.50 \%$ and $0.1 \%)$

NPIII: Nanoparticles of CS-TPP $(0.75 \%$ and $0.1 \%)$

NPIV: Nanoparticles of CS-TPP (1\% and $0.1 \%)$

Figure 13. Antibacterial activity of CS-TPP nanoparticles suspension against Staphylococcus aureus 


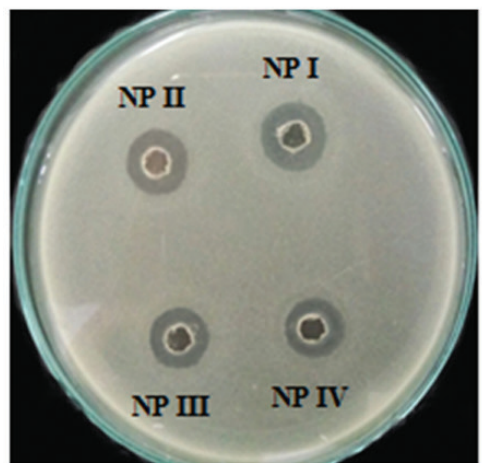

NPI : Nanoparticles of CS-TPP $(0.25 \%$ and $0.1 \%)$

NPII : Nanoparticles of CS-TPP $(0.50 \%$ and $0.1 \%)$

NPIII: Nanoparticles of CS-TPP $(0.75 \%$ and $0.1 \%)$

NPIV: Nanoparticles of CS-TPP (1\% and $0.1 \%)$

Figure 14. Antibacterial activity of CS-TPP nanoparticles suspension against Pseudomonas aeruginosa.

From Tables 2 and 3, we can see that with increasing CS concentrations from $0.25 \%$ to $1 \%$ and with TPP concentration $0.1 \%$, the antibacterial activity against Staphylococcus aureus and Pseudomonas aeruginosa increased that can be observed in the increasing of the diameter of ZOI. The inhibitory activity of Gram-positive is higher than in Gram-negative bacteria, however, it is not significantly different.

CS has an antibacterial activity because CS in acidic condition, $\mathrm{pH}$ below its $\mathrm{pKa}$ (6.3), the amino group of $\mathrm{CS}$ will be protonated into positively charged and produce $\mathrm{NH}_{3}^{+}$. This positively charged CS will interact with the negatively charged bacterial cell surface. In Gram-positive bacteria, the negative charge is caused by the cell wall that composed of peptidoglycan and teichoic acid, whereas in Gram-negative bacteria has an outer membrane composed of lipopolysaccharides and proteins. Therefore, the antibacterial activity of CS may occur due to the electrostatic interaction on the cell surface involving the cell wall or the outer membrane of the bacteria (Kong et al., 2010).

In Gram-positive bacteria, the teichoic acid will interact with the positive charge of CS on the cell surface and causes a disruption of membrane function (Raafat et al., 2008). In addition, the interaction between CS and the bacteria cell wall will also result in the accumulation of CS molecules on the surface of bacteria cells and inhibits the transport of nutrients into cells (Tokura et al., 1997). In Gram-negative bacteria, lipopolysaccharides and proteins present in the outer membrane are stabilized by electrostatic interaction with divalent cations. The existence of a polycation such as CS will compete with divalent cations to bind to the polyanion if the $\mathrm{pH}$ is below the $\mathrm{pKa}$ of CS; consequently, the permeability of the outer membrane of the bacterial cell will be disrupted and the cell membrane is damaged. Furthermore, the interactions may denature membrane proteins and initiate penetration into the phospholipid bilayer. The increased membrane permeability leads to destabilization of the cell membrane and leakage of intracellular substances; ultimately, the death of cells (Liu et al., 2004).

From Table 2, we can see that the antibacterial activity of nanoparticles suspension against Staphylococcus aureus is higher than the CS solution. Antibacterial activity against Pseudomonas aeruginosa also shown a similar result that is CS-TPP nanoparticles suspension gives higher activity than CS solution only. These results are due to nanoparticles providing more cationic site than CS that can interact with the negative charge of membrane cell bacteria. The inhibitory activity of
Gram positive is higher than in Gram-negative bacteria but is not significantly different.

From Table 1, we can see that with increasing CS concentrations, the particle size of CS-TPP nanoparticles increased. However, the smaller particle size shows the higher diameter of ZOI as shown in Tables 2 and 3, it means that the smaller particle gives higher antibacterial activity. The small particle size of nanoparticles suspension rendered them with unique physicochemical properties, such as the large surface area (providing more cationic sites) and high reactivity and thus could potentially enhance the charge interaction on the microbial surface and lead to more superior antimicrobial effect (Zhang et al., 2010). CS-TPP nanoparticles have a larger surface area so that nanoparticles can be tightly absorbed onto the surface of the bacteria cells so as to disrupt the membrane, which would lead to the leakage of intracellular components, thus killing the bacteria cells (Avadi et al., 2004; Qi et al., 2004).

\section{CONCLUSIONS}

Variation of CS and TPP concentrations is used to affect the particle size of nanoparticles suspension. The increase in CS concentration causes the particle size to increase. The best concentration of CS and TPP among formulation used to produce higher antibacterial activity of nanoparticles suspension is $0.25 \%$ and $0.1 \%$, respectively. The antibacterial activity of CS-TPP nanoparticles suspension is higher than that of the CS solution. The particle size of nanoparticles suspension plays an important role in the antibacterial activity where smaller particle size will show higher antibacterial activity.

\section{CONFLICT OF INTEREST}

The authors declare that they have no conflict of interest.

\section{REFERENCES}

Agnihotri SA, Nadagouda NM, Tejraj MA. Recent advances on chitosan based micro- and nanoparticles in drug delivery. J Control Release, 2004; 100:5-28.

Antoniou J, Fei L, Hamid M, Jing Q, Wallace Y, Fang Z. Physicochemical and morphological properties of size-controlled chitosantripolyphosphate nanoparticles. colloids and surface A: physicochem. Eng Aspects, 2015; 465:137-46.

Arianto A, Hakim B, Urip H, Syafruddin I. The comparison of swelling, mucoadhesive, and release of ranitidine from spherical matrices of alginate, chitosan, alginate-chitosan, and calcium alginate-chitosan. Int $\mathrm{J}$ PharmTech Res, 2014; 6(7):2054-63. 
Arianto A, Hakim B, Urip H, Syafruddin I. Effect of alginate chitosan ratio on the swelling, mucoadhesive, and release of ranitidine from spherical matrices of alginate-chitosan. Int J PharmTech Res, 2015; 8(4):653-65.

Avadi M, Sadeghi A, Tahzibi A, Bayati K, Pouladzadeh M, Zohuriaan-Mehr M, Rafiee-Tehrani M. Diethylmethyl chitosan as an antimicrobial agent: synthesis, characterization and antibacterial effects. Euro Polym J, 2004; 40(7):1355-61.

Aziz MA, Jaydee DC, Heather JLB, Stephen CM, Lyall RH. Antimicrobial properties of a chitosan dextran-based hydrogel for surgical use. Antimicrob Agents Chemother, 2012; 56(1):280-7.

Calvo P, Remunan-Lopez C, Vila-Jato JL, Alonso MJ. Novel hydrophilic chitosan-polyethylene oxide nanoparticles as protein carriers. J Appl Polym Sci, 1997a; 63:125-32.

Calvo P, Vila-Jato JL, Maria JA. Evaluation of cationic polymercoated nanocapsules as ocular drug carriers. Int J Pharm, 1997b; 153:41-50.

Chen DB, Tian ZY, Wang LL, Qiang Z. In vitro and in vivo study of two types of long-circulating solid lipid nanoparticles containing paclitaxel. Chem Pharm Bull, 2001; 49(11):1444-7.

Dai T, Masamitsu T, Ying YH, Michael RH. Chitosan preparation for wounds and burns: antimicrobial and wound healing effects. Expert Rev Anti Infect Ther, 2011; 9(7):857-79.

Florence D. Evaluation of nano and microparticle uptake by the gastrointestinal tract. Adv Drug Deliv Rev, 1998; 34:221-33.

Ibrahim HM, Manal KEB, Ghada MT, Ekhlas AEA. Chitosan nanoparticles loaded antibiotics as drug delivery biomaterial. J Appl Pharm Sci, 2015; 5(10):85-90.

Illum L. Chitosan and its use as a pharmaceutical excipient. Pharm Res, 1998; 15:1326-31.

Jafary F, Panjehpour M, Varshosaz J, Yaghmaei P. Stability improvement of immobilized alkaline phosphatase using chitosan nanoparticles. Brazil J Chem Eng, 2016; 33(2):243-50.

Kayser O, Lemke A, Hernandez-Trejo N. The impact of nanobiotechnology on the development of new drug delivery systems. Curr Pharm Biotechnol, 2005; 6(1):3-5.

Kong M, Xi GC, Ke X, Hyun JP. Antimicrobial properties of chitosan and mode of action: a state of the art review. Int J Food Microbiol, $2010 ; 144: 51-63$.

Kumar DA, Singh D, Mishra J, Nirwan S, Pandey SP. Development and characterization of chitosan nanoparticles loaded with amoxycillin. Int Res J Pharm, 2011; 2(5):145-51.

Leceta I, Guerrero P, Ibarburu, Duenas MT, de la Caba K. Characterization and antimicrobial analysis of chitosan-based films. J Food Eng, 2013; 116:889-99.

Liu H, Yumin D, Xiaohui W, Liping S. Chitosan kills bacteria through cell membrane damage. Int J Food Microbiol, 2004; 95:147-55.
Mariadi HB. Karsono: Formulation and in vitro evaluation of gastroretentive drug delivery system of antacids using alginate-chitosan films. Int J PharmTech Res, 2015; 8(9):1-12.

Mohanraj VJ, Chen Y. Nanoparticles - a review. Trop J Pharm Res, 2006; 5(1):561-73.

Nagarajan E, Shanmugasundaram $\mathrm{P}$, Ravichandiran V, Vijayalakshmi A, Senthilnathan B, Masilamani K. Development and evaluation of chitosan based polymeric nanoparticles of an antiulcer drug lansoprazole. J Appl Pharm Sci, 2015; 5(4):20-5.

Nie J, Zhengke W, Kai Z, Qiaoling H. Biomimetic multilayered hollow chitosan-tripolyphosphate rod with excellent mechanical performance. RSC Adv, 2015; 47:1-7.

Prabaharan M, Mano JF. Chitosan-based particles as controlled drug delivery systems. Drug Deliv, 2005; 12(1):41-57.

Qi L, Zirong X, Xia J, Caihong H, Xiangfei Z. Preparation and antibacterial activity of chitosan nanoparticles. Carbohydr Res, 2004 339:2693-700.

Raafat D, Kristine VB, Albert H, Hans GS. Insights into the mode of action of chitosan as an antibacterial compound. Appl Environ Microbiol, 2008; 74(12):3764-73.

Shu XZ, Zhu KJ. Controlled drug release properties of ionically cross-linked chitosan beads: the influence of anion structure. Int J Pharm, 2002; 223(1-2):217-25.

Singh D, Shashi A, Alok M, Kaushal K. Advancement of chitosan-based nanoparticles for targeted drug delivery of antiulcer drugs. World J Pharm Pharm Sci, 2015; 4(1):505-18.

Tokura S, Keisuke U, Satoshi M, Norio N. Molecular weight dependent antimicrobial activity by chitosan. Macromol Symp, 1997; 120:1-9.

Yasmeen S, Mrinal KK, Badhan S, Md. Rakibul Qadir, Md. Abdul Gafur, Shah MM. Chromium (VI) ions removal from tannery effluent using chitosan-microcrystalline cellulose composite as adsorbent. Int Res J Pure Appl Chem, 2016; 10(4):1-14.

Yongmei X, Yumin D. Effect of molecular structure of chitosan on protein delivery properties of chitosan nanoparticles. Int J Pharm, 2003; 250:215-26.

Zhang L, Pornpattananangkul $\mathrm{D}$, $\mathrm{Hu}$ CMJ, Huang CM Development of nanoparticles for antimicrobial drug delivery. Curr Med Chem, 2010; 17(6):585-94.

How to cite this article:

Bangun H, Tandiono S, Arianto A. Preparation and evaluation of chitosan-tripolyphosphate nanoparticles suspension as an antibacterial agent. J App Pharm Sci, 2018; 8(12): 147-156. 